\title{
Excess placental secreted frizzled-related protein 1 in maternal smokers impairs fetal growth
}

\author{
Alice Wang, ${ }^{1,2}$ Zsuzsanna K. Zsengellér, ${ }^{2}$ Jonathan L. Hecht, ${ }^{2}$ Roberto Buccafusca, ${ }^{2}$ Suzanne D. Burke, ${ }^{2}$ Augustine Rajakumar, ${ }^{2}$ \\ Emily Weingart, ${ }^{3}$ Paul B. Yu, ${ }^{4}$ Saira Salahuddin, ${ }^{2}$ and S. Ananth Karumanchi ${ }^{2}$
}

'Boston University Medical Center, Boston, Massachusetts, USA. ${ }^{2}$ Beth Israel Deaconess Medical Center, Boston, Massachusetts, USA. ${ }^{3}$ Mercy College, New York, New York, USA.

${ }^{4}$ Brigham and Women's Hospital, Boston, Massachusetts, USA.

\begin{abstract}
Maternal cigarette smoking during pregnancy remains one of the most common and preventable causes of fetal growth restriction (FGR), a condition in which a fetus is unable to achieve its genetically determined potential size. Even though epidemiologic evidence clearly links maternal cigarette smoking with FGR, insight into the molecular mechanisms of cigarette smoke-induced FGR is lacking. Here, we performed transcriptional profiling of placentas obtained from smoking mothers who delivered growth-restricted infants and identified secreted frizzled-related protein 1 (sFRP1), an extracellular antagonist of endogenous WNT signaling, as a candidate molecule. sFRP1 mRNA and protein levels were markedly upregulated ( 10fold) in placentas from smoking mothers compared with those from nonsmokers. In pregnant mice, adenovirus-mediated overexpression of sFRP1 led to FGR, increased karyorrhexis in the junctional zone, and decreased proliferation of labyrinthine trophoblasts. Consistent with our hypothesis that placental WNT signaling is suppressed in maternal smokers, we found that exposure to carbon monoxide analogs led to reduced WNT signaling, increased SFRP1 mRNA expression, and decreased cellular proliferation in a trophoblast cell line. Moreover, administration of carbon monoxide analogs to pregnant mice in late gestation led to FGR. In summary, our results indicate that the increased placental expression of sFRP1 seen in smokers impairs fetal growth by inhibiting WNT signaling and trophoblast proliferation.
\end{abstract}

\section{Introduction}

Fetal growth restriction (FGR), a condition in which a fetus is unable to achieve its genetically determined potential size, results in substantial perinatal and neonatal morbidity and mortality (1). Infants of mothers who smoke weigh on average 200-400 grams less than gestational age-matched controls (2), with the degree of fetal weight reduction relating directly to the quantity of smoke exposure (3). Size at birth also affects adult rates of mortality and morbidity $(1,4,5)$. Chronic exposure of the fetus to the effects of tobacco smoke remains the most important preventable risk factor for FGR.

The cellular targets of gestational exposure to tobacco products are not known; however, the deleterious effects of smoking on fetal growth are thought to be mediated primarily by effects on the placenta, given the changes seen in histomorphometric studies of placentas from smoking mothers (6-11). Although the association of maternal cigarette smoking with FGR is well established, the mechanism by which smoking alters placental architecture is poorly understood, primarily because the molecular mechanisms regulating trophoblast proliferation and differentiation have not been fully elucidated.

To gain insight into how cigarette smoking can affect placental development and function, we performed an unbiased screen of genes in placentas from pregnancies affected by FGR and found

Conflict of interest: The authors have declared that no conflict of interest exists. Submitted: December 11, 2014; Accepted: August 17, 2015

Reference information: / Clin Invest. 2015;125(11):4021-4025. doi:10.1172/JCI80457. marked dysregulation of the WNT/T cell-specific factor (WNT/ TCF) signaling family. The WNT signaling family consists of a large family of highly conserved, secreted, glycosylated ligands known to be critical for embryonic development, cell proliferation, and fate determination, especially in progenitor cell populations critical for organ renewal $(12,13)$. Here, we report that placental upregulation of secreted frizzled-related protein 1 (sFRP1), a WNT antagonist (14) seen in maternal smokers, leads to FGR.

\section{Results and Discussion}

Smoking mothers have elevated placental gene and protein levels of $s F R P 1$. To evaluate novel pathways that regulate fetal growth affected by mothers who smoke, we isolated placental RNA from smoking mothers with severe FGR and compared it by microarray analysis to that of nonsmoking mothers with appropriately grown fetuses. Analysis of the top upregulated and downregulated genes (Supplemental Figure 1A; supplemental material available online with this article; doi:10.1172/JCI80457DS1) revealed dramatic upregulation of SFRP1 mRNA in placentas from smoking mothers. Quantitative RT-PCR analysis of placental mRNA of a larger cohort of placentas from smoking mothers $(n=7)$ and nonsmoking mothers $(n=14)$ confirmed a $>10$-fold upregulation of SFRP1 in smokers (Figure 1A). sFRP1 protein expression levels were also found to be $>10$-fold higher in placentas exposed to maternal smoking $(n=9)$ compared with placentas of nonsmoking controls $(n=8)$ by immunoblot analysis (Figure 1, B and C). Pregnancies complicated by smoking-related FGR $(n=3)$ had significantly increased placental 

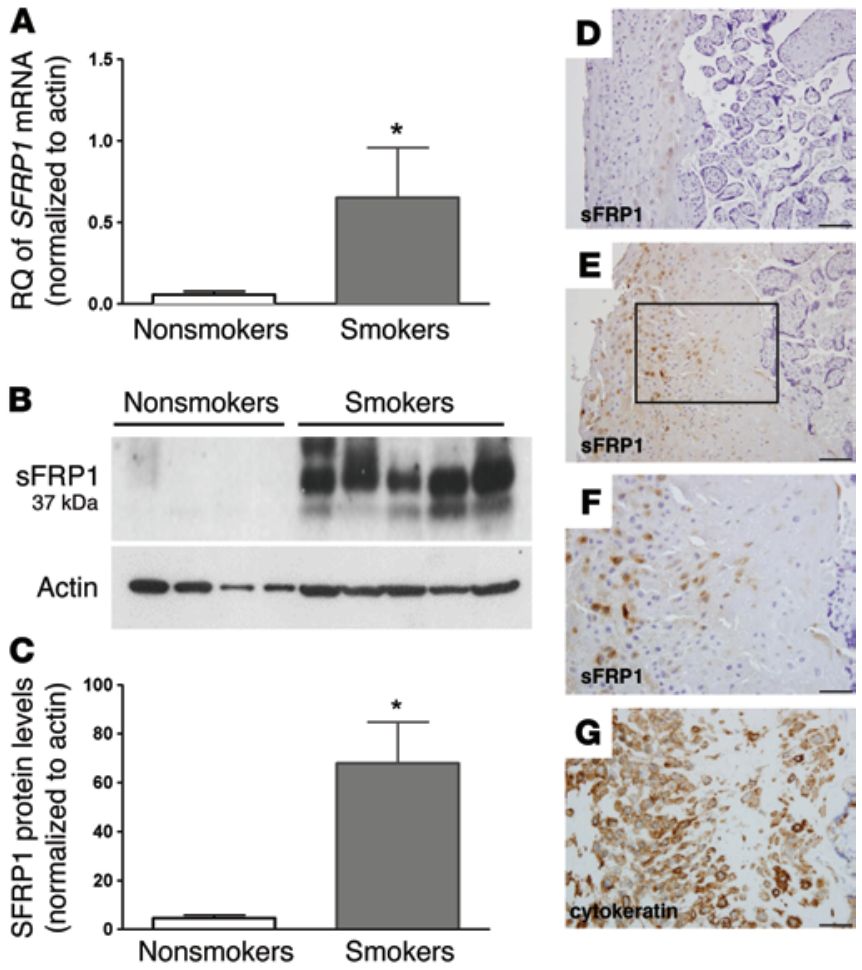

SFRP1 gene expression compared with pregnancies with preeclampsia-associated FGR $(n=3)$ and control pregnancies with gestational age-matched appropriate for gestational age (AGA) fetuses $(n=6)$ (Supplemental Figure 1B).

sFRP1 belongs to a family of soluble proteins called secreted frizzled-related proteins (sFRPs) that are important modulators of WNT signaling (15). sFRPs function as WNT antagonists by trapping WNT ligands or by binding Frizzled to prevent the interaction of WNT ligands with their receptors (16). By immunohistochemistry staining for cytokeratin and sFRP1, sFRP1 appeared to be localized to invasive extravillous trophoblasts (Figure $1, D-G$ ). In addition, consistent with previously published studies $(17,18)$, placentas from smoking mothers showed evidence of significantly decreased proliferative activity, as shown by Ki67 staining in cytotrophoblasts (Supplemental Figure 1, C and D). Taken together with prior gene-targeting experiments that suggest a role for WNT signaling in murine placental development (19-21), the present data support the concept that elevated placental expression of sFRP1 may lead to abnormalities in placentation.

sFRP1 overexpression in pregnant mice leads to FGR. While transgenic mice that globally overexpress the SFRP1 gene have been reported to be protected from cardiac rupture and myocardial infarction (22), analysis of fetal growth and placental function of the transgenic fetuses and transgenic mothers have not been reported. To specify a limited window of sFRP1 overexpression during pregnancy and to evaluate the significance of a circulating form of SFRP1, we created a truncated construct of sFRP1 lacking the heparin-binding domain and used a model of adenovirus-mediated gene transfer. Pregnant dams were injected with an adenovirus expressing a truncated version of sFRP1 (Supplemental Figure 2A) to induce expression of the protein in the systemic circulation. Expression of the soluble
Figure 1. Elevated SFRP1 gene and protein expression in the placentas of smokers. (A) Relative quantification (RQ) of SFRP1 mRNA levels measured, as by qRT-PCR of placentas from smoking mothers $(n=7)$ and nonsmoking mothers $(n=14)$ (mean $\pm \mathrm{SEM},{ }^{*} P<0.05$ versus nonsmoking controls by $t$ test). (B) Representative immunoblot of sFRP1 and $\beta$-actin protein expression in placental extracts obtained from nonsmoking and smoking mothers. (C) Quantification of immunoblots by densitometry of sFRP1 and $\beta$-actin protein expression in placental extracts obtained from nonsmoking $(n=8)$ and smoking $(n=9)$ mothers (mean \pm SEM, ${ }^{*} P<0.001$ versus nonsmoking controls by $t$ test). (D-G) Representative images of human placental tissue sections with immunostaining for anti-sFRP1 from (D) a nonsmoker and (E) a smoker. Serial placental sections from a smoker with immunostaining (F) for anti-sFRP1 and (G) for anti-cytokeratin show localization of SFRP1 to the extravillous trophoblast. The boxed region in $\mathbf{E}$ is shown at high magnification in $\mathbf{F}$ and $\mathbf{G}$. Five placentas from smokers and five placentas from nonsmokers were compared. Scale bar: $100 \mu \mathrm{m}$ (D and $\mathbf{E}) ; 50 \mu \mathrm{m}$ (F and $\mathbf{G}$ ).

truncated sFRP1 in circulation was confirmed by immunoblot (Supplemental Figure 2B). After injection on E15, a time comparable to the human third trimester when the most rapid fetal growth occurs, fetuses from sFRP1 virus-treated dams $(n=7$ litters, $n=77$ fetuses) had a mean weight reduction of $149 \mathrm{mg}$, or $10.5 \%$, compared with that of control virus-treated dams $(n$ $=9$ litters, $n=120$ fetuses) (Figure 2A). The $10 \%$ reduction in fetal weights was consistent with the reported average birth weight differences between smokers and nonsmokers (2). Placental weights on E19 were unchanged (Figure 2B), consistent with epidemiological studies that reported no differences in mean placental weight in maternal smokers compared with nonsmokers $(10,23)$. Histological sections of murine placentas demonstrated increased spongiotrophoblast karyorrhexis in the junctional zone of sFRP1 virus-treated fetuses compared with control placentas (Figure 2C). Ki67 staining, a marker of trophoblast proliferation, was $38 \%$ lower in the placentas of sFRP1 virus-treated animals compared with that in control placentas (Figure 2, D and E). Consistent with human epidemiological studies that demonstrate that the most deleterious effects of cigarette smoking occur in the last trimester of pregnancy $(3,24)$, elevated levels of sFRP1 by adenoviral overexpression appeared to have marked effects upon fetal growth when introduced during late gestation in mice, with increased karyorrhexis and decreased trophoblast proliferation noted in the placentas.

Effect of carbon monoxide analogs on trophoblast proliferation and fetal growth. Transcriptional activity of the canonical WNT signaling pathway was examined following transient expression of a TCF reporter plasmid in both HEK293 cells (Supplemental Figure 3) and the human trophoblast line HTR-8/SVneo (Figure $3 \mathrm{~A}$ ), with or without exposure to varying concentrations of cigarette smoke extract, carbon monoxide-releasing molecule 
A

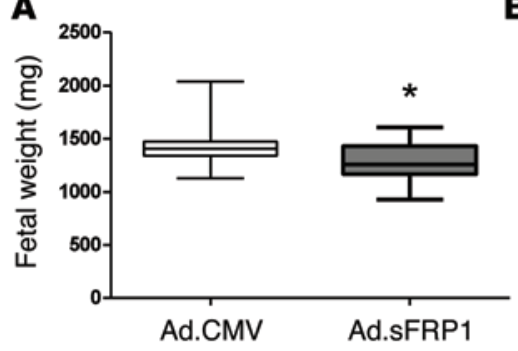

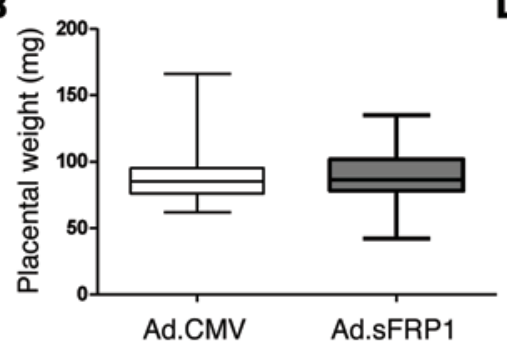

D


C
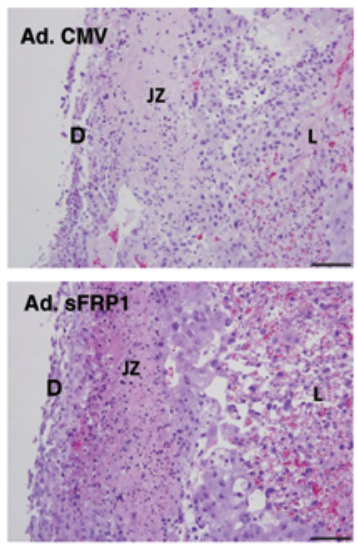
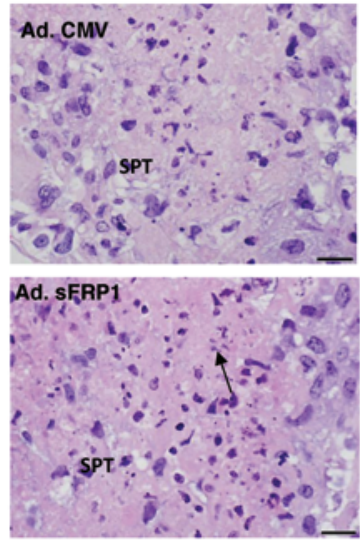

\author{
BRIEF REPORT
}


A
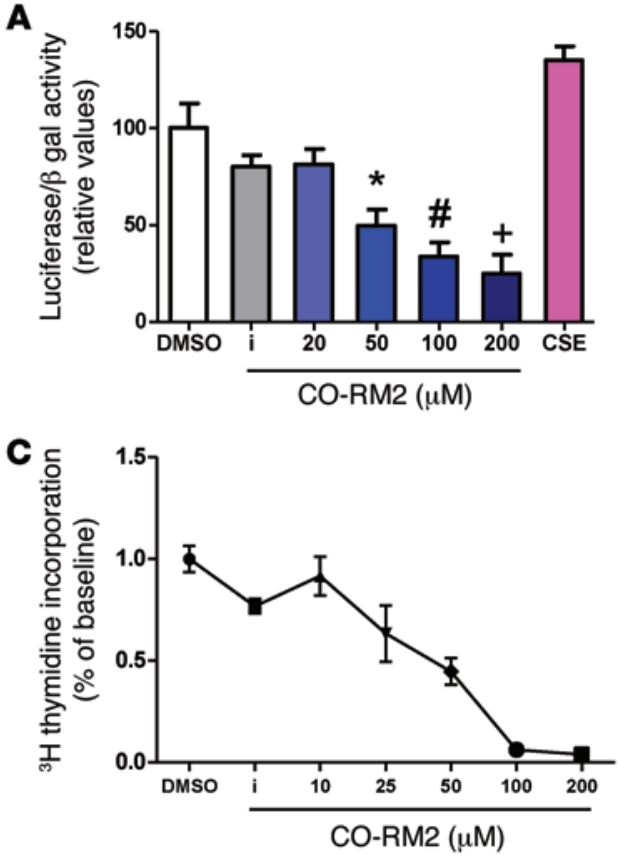

B

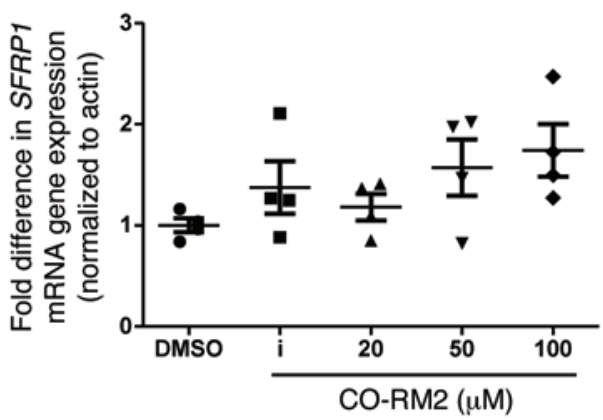

D

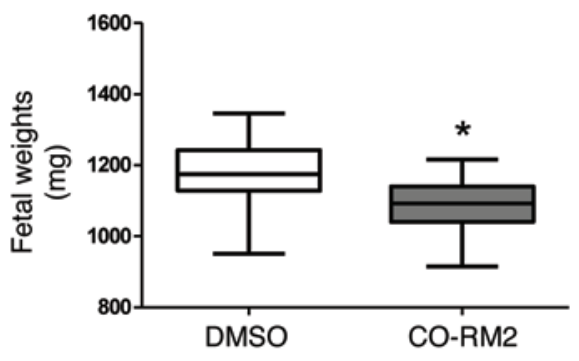

Figure 3. Effect of carbon monoxide analogs on canonical WNT transcriptional activity, sFRP1 expression, trophoblast proliferation, and fetal growth. (A) Luciferase activity of the canonical WNT reporter in HTR cells transfected with the TOPFLASH TCF reporter plasmid after exposure to varying concentrations of CO-RM2, inactivated CO-RM2 (iCO-RM2), and 10\% cigarette smoke extract (CSE) for a period of 24 hours. The normalized value of unstimulated control was arbitrarily set at $100 \%$. Bars represent mean values of 3 different experiments; error bars indicate SEM. $P<0.001$ by ANOVA, ${ }^{*} P<0.05$ for $50 \mu \mathrm{M}$ CO-RM2 versus DMSO control by $t$ test, ${ }^{\#} P<0.05$ for $100 \mu \mathrm{M}$ CO-RM2 versus control by $t$ test, ${ }^{+} P<0.05$ for $200 \mu \mathrm{M}$ CO-RM2 versus DMSO control by $t$ test. (B) Relative quantification of SFRP1 mRNA levels measured by qRT-PCR in HTR cells after exposure to CO-RM2 for a period of 24 hours. Data are presented as scatter plot (mean \pm SEM). SFRP1 expression was increased after exposure to $100 \mu \mathrm{M} C 0-\mathrm{RM} 2$ compared with that after exposure to DMSO control $\left(P<0.05\right.$ by $t$ test). (C) Dose-dependent reduction of $\left[{ }^{3} \mathrm{H}\right]$ thymidine incorporation in serum-starved HTR cells after exposure to varying concentrations of CO-RM2. Inactivated $\mathrm{CO}-\mathrm{RM} 2$ had no effect of $\left[{ }^{3} \mathrm{H}\right]$ thymidine incorporation. Experiments were performed twice, in triplicate (mean \pm SEM). (D) Fetal weights on E18 were decreased in CD1 pregnant mice injected on E15 with CO-RM2 ( $n=5$ litters, 63 fetuses) as compared with those injected with $1 \%$ DMSO vehicle ( $n=5$ litters, 67 fetuses). Data are presented as a box plot (25th-75th percentile), with horizontal bars representing median values and whiskers representing minimum and maximum values ( ${ }^{*} P<0.001$ versus mice injected with DMSO vehicle by $t$ test).

\section{Methods}

Human studies. Placental tissue specimens were obtained immediately after vaginal or cesarean delivery. Subjects were divided into two groups (maternal cigarette smoking exposure and nonsmoking control) (Supplemental Table 1). The degree of maternal smoking exposure was obtained from self-reported information obtained from the medical chart. Any amount of regular cigarette use during pregnancy was considered to be active smoking. Smokers were then characterized as smoking FGR or smoking AGA. Nonsmoking pregnant women with gestational agematched, AGA fetuses and nonsmoking women with preeclampsia-associated FGR were included as controls. FGR was defined as having a birthweight below the fifth percentile based on the Alexander criteria (31).

Mouse studies. Timed pregnant CD1 mice were injected intravenously at E15 with adenovirus expressing sFRP1 or a control (CMV-null) virus ( $n=7-9$ pregnant mice per group), and phenotypes were evaluated at E19. Alternatively, animals were injected with CO-RM2 or 1\% DMSO vehicle ( $n=5$ mice per group) during the same gestational window.

Transcriptome data. Transcriptome data were deposited in the NCBI's Gene Expression Omnibus (accession no. GSE71931).

Supplemental Methods. Detailed protocols for other methods used are described in the Supplemental Methods.

Statistics. Statistical analyses were performed using Prism 6 software (Graphpad). A 2-tailed Student's $t$ test was used to analyze control and diseased or treatment groups. In analyzing fetal weights, each individual fetal weight was considered a biological replicate. Comparisons between multiple groups were made by ANOVA with Dunn's post-test, with $P$ values of less than 0.05 being considered significant.

Study approval. Human studies were approved by the institutional review boards at Beth Israel Deaconess Medical Center (BIDMC) and Boston University Medical Center. All animal studies were approved by the BIDMC Institutional Animal Care and Use Committee and were in accordance with the NIH's Guide for the Care and Use of Laboratory Animals (8th ed. The National Academies Press. 2011.).

\section{Acknowledgments}

A. Wang was supported by a Young Clinical Scientist Award from the Flight Attendant Medical Research Institute and Wing Tat Lee pilot funding from Boston University School of Medicine. S.D. Burke and S.A. Karumanchi were supported by the Howard Hughes Medical Institute. We thank Manoj Bhasin at the BIDMC Genomics Core Facility for help with microarray analysis.

Address correspondence to: Alice Wang, Boston University Medical Center, 771 Albany Street, Dowling 4109, Boston, Massachusetts 02118, USA. Phone: 617.414.3697; E-mail: alice.wang@bmc.org. Or to: S. Ananth Karumanchi, Beth Israel Deaconess Medical Center, 330 Brookline Ave., RN 370D, Boston, Massachusetts 02215, USA. Phone: 617.667.1018; E-mail: sananth@bidmc.harvard.edu. 
1. Andres RL, Day MC. Perinatal complications associated with maternal tobacco use. Semin Neonatol. 2000;5(3):231-241.

2. Wang X, et al. Maternal cigarette smoking, metabolic gene polymorphism, and infant birth weight. JAMA. 2002;287(2):195-202.

3. Bernstein IM, Mongeon JA, Badger GJ, Solomon L, Heil SH, Higgins ST. Maternal smoking and its association with birth weight. Obstet Gynecol. 2005;106(5 pt 1):986-991.

4. Barker DJ. Outcome of low birthweight. Horm Res. 1994;42(4-5):223-230.

5. Barker DJ, Fall CH. Fetal and infant origins of cardiovascular disease. Arch Dis Child. 1993;68(6):797-799.

6. Klesges LM, Murray DM, Brown JE, Cliver SP, Goldenberg RL. Relations of cigarette smoking and dietary antioxidants with placental calcification. Am J Epidemiol. 1998;147(2):127-135.

7. Carmines EL, Gaworski CL, Faqi AS, Rajendran $\mathrm{N}$. In utero exposure to $1 \mathrm{R} 4 \mathrm{~F}$ reference cigarette smoke: evaluation of developmental toxicity. Toxicol Sci. 2003;75(1):134-147.

8. Genbacev O, Bass KE, Joslin RJ, Fisher SJ. Maternal smoking inhibits early human cytotrophoblast differentiation. Reprod Toxicol. 1995;9(3):245-255.

9. Burton GJ, Palmer ME, Dalton KJ. Morphometric differences between the placental vasculature of non-smokers, smokers and ex-smokers. $\mathrm{Br} \mathrm{J}$ Obstet Gynaecol. 1989;96(8):907-915.

10. Larsen LG, Clausen HV, Jonsson L. Stereologic examination of placentas from mothers who smoke during pregnancy. Am JObstet Gynecol. 2002;186(3):531-537.

11. Bush PG, Mayhew TM, Abramovich DR, Aggett PJ, Burke MD, Page KR. Maternal cigarette smoking and oxygen diffusion across the placenta. Placenta. 2000;21(8):824-833.

12. Clevers $H$, Nusse R. Wnt/ $\beta$-catenin signaling and disease. Cell. 2012;149(6):1192-1205.

13. Wang B, Zhao L, Fish M, Logan CY, Nusse R. Self-renewing diploid Axin2 cells fuel homeostatic renewal of the liver. Nature. 2015;524(7564):180-185.

14. Duplaa C, Jaspard B, Moreau C, D’Amore PA. Identification and cloning of a secreted protein related to the cysteine-rich domain of frizzled. Evidence for a role in endothelial cell growth control. Circ Res. 1999;84(12):1433-1445.

15. Bovolenta P, Esteve P, Ruiz JM, Cisneros E, LopezRios J. Beyond Wnt inhibition: new functions of secreted frizzled-related proteins in development and disease. J Cell Sci. 2008;121(pt 6):737-746.

16. Rodriguez J, et al. SFRP1 regulates the growth of retinal ganglion cell axons through the Fz2 receptor. Nat Neurosci. 2005;8(10):1301-1309.

17. Arnholdt H, Meisel F, Fandrey K, Lohrs U. Proliferation of villous trophoblast of the human placenta in normal and abnormal pregnancies. Virchows Arch B Cell Pathol Incl Mol Pathol.1991;60(6):365-372.

18. Genbacev O, et al. Concordant in situ and in vitro data show that maternal cigarette smoking negatively regulates placental cytotrophoblast passage through the cell cycle. Reprod Toxicol. 2000;14(6):495-506.

19. Monkley SJ, Delaney SJ, Pennisi DJ, Christiansen JH, Wainwright BJ. Targeted disruption of the Wnt2 gene results in placentation defects. Development. 1996;122(11):3343-3353.

20. Parr BA, Cornish VA, Cybulsky MI, McMahon AP. Wnt7b regulates placental development in mice. Dev Biol. 2001;237(2):324-332.

21. Galceran J, Farinas I, Depew MJ, Clevers H, Grosschedl R. Wnt3a-/--like phenotype and limb deficiency in Lef1(-/-)Tcf1(-/-) mice. Genes Dev. 1999;13(6):709-717.

22. Barandon L, et al. Reduction of infarct size and prevention of cardiac rupture in transgenic mice overexpressing FrzA. Circulation.
2003;108(18):2282-2289.

23. van der Velde WJ, Treffers PE. Smoking in pregnancy: the influence on percentile birth weight, mean birth weight, placental weight, menstrual age, perinatal mortality and maternal diastolic blood pressure. Gynecol Obstet Invest. 1985;19(2):57-63.

24. Lieberman E, Gremy I, Lang JM, Cohen AP. Low birthweight at term and the timing of fetal exposure to maternal smoking. Am J Public Health. 1994;84(7):1127-1131.

25. Sonderegger S, et al. Wingless (Wnt)-3A induces trophoblast migration and matrix metalloproteinase- 2 secretion through canonical Wnt signaling and protein kinase B/AKT activation. Endocrinology. 2010;151(1):211-220.

26. Carmines EL, Rajendran N. Evidence for carbon monoxide as the major factor contributing to lower fetal weights in rats exposed to cigarette smoke. Toxicol Sci. 2008;102(2):383-391.

27. Foronjy R, et al. The divergent roles of secreted frizzled related protein-1 (SFRP1) in lung morphogenesis and emphysema. Am J Pathol. 2010;177(2):598-607.

28. Blacquière MJ, Timens W, van den Berg A, Geerlings M, Postma DS, Hylkema MN. Maternal smoking during pregnancy decreases Wnt signalling in neonatal mice. Thorax. 2010;65(6):553-554

29. Marsit CJ, et al. Epigenetic inactivation of SFRP genes and TP53 alteration act jointly as markers of invasive bladder cancer. Cancer Res. 2005;65(16):7081-7085.

30. Schiefer L, et al. Epigenetic regulation of the secreted frizzled-related protein family in human glioblastoma multiforme. Cancer Gene Ther. 2014;21(7):297-303.

31. Zhang J, Bowes WA Jr. Birth-weight-for-gestational-age patterns by race, sex, and parity in the United States population. Obstet Gynecol. 1995;86(2):200-208. 